\title{
El aprendizaje significativo y su relación con los estilos de aprendizaje
}

\author{
Luis Fernando Garcés Cobos \\ Secretaría de Educación, Ciencia, Tecnología e Innovación - SENESCYT \\ fgarces0402@hotmail.com \\ Ángel Montaluisa Vivas \\ Universidad Central del Ecuador \\ aemontaluisa@uce.edu.ec \\ Edgar Salas Jaramillo \\ Universidad Central del Ecuador \\ esalasj2003@yahoo.com.ar
}

Recibido: 30 - noviembre - 2018 / Aprobado: 21 - diciembre - 2018

\section{Resumen}

Este trabajo tiene el propósito de aportar algunos conocimientos teóricos del aprendizaje significativo de Ausubel y sus relaciones con el modelo de estilos de aprendizaje de Honey y Mumford. En este sentido, se repasa las características más relevantes, requerimientos y factores necesarios del aprendizaje significativo, para lograr el proceso de asimilación de la información en la estructura cognitiva, es decir, los estudiantes deben ser capaces de desarrollar habilidades y destrezas propias. Después se hace un análisis de los estilos de aprendizaje -los modelos de Kolb y Honey Mumford-, y su influencia en el proceso de enseñanza y aprendizaje. Finalmente, se concluye que el docente debe conocer los estilos de aprendizaje y preferencias individuales de sus alumnos para programar actividades metodológicas y actividades que conecten la estructura cognitiva de una disciplina con la previa, pues no hay ningún aprendizaje que empiece de cero. 
Palabras clave: aprendizaje significativo, estructura cognitiva, asimilación cognitiva, constructivismo, estilos de aprendizaje.

\section{Abstract}

This paper aims to provide some theoretical knowledge of meaningful learning of Ausubel and its relations with the model of learning styles Honey and Mumford. In this sense, the most relevant characteristics, requirements and necessary factors of meaningful learning is reviewed, to achieve the process of assimilating information in cognitive structure, ie, students should be able to develop abilities and skills. After an analysis of learning styles models -the Kolb and Honey Mumford, and its influence on the process of teaching and learning is done. Finally, we conclude that the teacher should know the learning styles and preferences of students to schedule methodological activities and activities that connect the cognitive structure of a prior discipline because there is no learning start from scratch.

Keywords: significant learning, cognitive structure, cognitive assimilation, constructivism, learning styles. 


\section{Introducción}

$\mathrm{E}$ n un mundo globalizado, el ser humano enfrenta procesos de integración histórica, económica, política y educativa. Es así como, “aprender a aprender" es una opción que va a convertirse en una de las capacidades de supervivencia social. El problema fundamental dentro de este estudio es cómo el aprendizaje significativo está relacionado directamente con los estilos de aprendizaje y su incidencia en el proceso de enseńanza. Es por esto, que resaltan cuestiones como: ¿Es posible que el aprendizaje significativo trascienda? ¿Se pueden relacionar el aprendizaje significativo con los estilos de aprendizaje propuesto por el modelo de Honey y Mumford?, ¿Qué factores intervienen en el aprendizaje memorístico o por descubrimiento? ¿Qué requerimientos son necesarios para desarrollar un aprendizaje significativo de calidad?

Este estudio es un análisis bibliográfico de tipo documental de investigaciones realizadas por David Ausubel y la propuesta de estilos de aprendizaje de Honey y Mumford (1986), quienes crean un cuestionario de cuatro estilos de aprendizaje: activo, reflexivo, teórico y pragmático. Más tarde adaptado por Catalina Alonso (1992), quien lo ajustó al español con el nombre de CHAEA, basándose en los resultados obtenidos en sus investigaciones, por lo que elaboró una lista con características que determinan el campo de destrezas de cada estilo.

En este contexto, el propósito de este artículo es contribuir con información útil para el conocimiento del aprendizaje significativo y los estilos de aprendizaje como requisitos necesarios en el proceso de enseńanza y aprendizaje.

Desde el punto de vista argumentativo, en primer lugar, se revisa la trascendencia y asimilación de la información del aprendizaje significativo, exigencias que se refieren a desarrollar la memoria a largo plazo, pues es un aprendizaje intrapersonal que interactúa directamente con las estructuras intelectuales de los individuos. En segundo lugar, la motivación, en el sentido de enfrentar los retos del aprendizaje, a través de la participación, autonomía y confianza en sí mismo, elementos importantes para procesar los conocimientos: requerimientos necesarios para aprender a conocer, aprender a hacer, aprender a vivir juntos y aprender a ser (Delors, 1994).

En otro instante, se analiza la tesis de que el aprendizaje significativo y su relación con los estilos de aprendizaje, especialmente, reflexionar sobre el modelo de Honey y Mumford, para diferenciar cómo los dicentes perciben, interaccionan y responden al aprendizaje significativo. La afirmación que todas las personas aprenden de una sola manera es una conjetura equivocada; ya que, es fácil evidenciar cómo cada uno prefiere un ambiente específico, la aplicación de diferentes metodologías, una situación contextual, un determinado tipo de ejercicios; es decir, se puede inferir que existen diferentes estilos y ritmos de aprendizaje.

Finalmente, se trata de reflexionar sobre los diferentes pensamientos teóricos (memorístico y por descubrimiento) que plantea el aprendizaje significativo, 
estableciendo relaciones conceptuales entre el aprendizaje significativo y el modelo propuesto por Honey y Mumford. Modelos que ayudan a comprender cómo el ser humano aprende a partir de los diferentes estilos, para ello elabora sus propias estrategias que tratan de explicar cómo los sujetos acceden al conocimiento significativo.

\section{El aprendizaje significativo: trascendencia y asimilación}

Para Kant, la trascendencia es la capacidad de la razón para acceder a un conocimiento superior de los objetos del mundo, es decir, plantea la superación de las fronteras del conocimiento, sobrepasando los límites cognoscitivos; por lo que, el propósito de trascender no es que el sujeto se acerque al objeto de estudio, sino el modo de conocer los conocimientos científicos (a priori) para aprender; de ahí que, un aprendizaje trasciende cuando tiene la característica de ser permanente $y$, por consiguiente, imperecedero. Dicho de otra manera, la trascendencia se da en el momento que el docente planifica tareas y actividades, para que el estudiante sea capaz de desarrollar habilidades y destrezas de aprendizaje propias, para luego ser replicadas en diferentes situaciones, Por ejemplo, facilitar el sentido de competencias (conocimientos), regulación de la conducta y control del comportamiento, desarrollar el sentido de compartir (Ortiz, 2010).

En este contexto, Ausubel, Novak y Hanesian (1983) se basa en el constructivismo para desarrollar la teoría del aprendizaje significativo. De acuerdo a esto, este aprendizaje se facilita cuando la nueva información se incorpora a estructura cognitiva del estudiante, provocando un proceso de asimilación cognoscitiva, en el que se relaciona la nueva información con los conocimientos previos. Es decir, el docente debe convertirse en un facilitador entre los conocimientos y los estudiantes a partir de actividades planificadas y organizadas. Lo importante es tratar de explicar ¿Cómo aprenden las personas? y ¿Por qué se olvidan lo que aprenden?

Según Ballester (2002), el aprendizaje significativo se desarrolla a largo plazo, es un procedimiento de contraste, de modificación de los esquemas de conocimiento, de equilibrio, de conflicto y de nuevo equilibrio otra vez. Es decir, es un proceso del aprendizaje, cuya finalidad es construir un equilibrio entre los conocimientos y la estructura cognitiva del individuo a partir de la nueva información obtenida, la cual puede ser modificada o transformada.

Para Ausubel (2002), el aprendizaje significativo se caracteriza por edificar los conocimientos de forma armónica y coherente, por lo que es un aprendizaje que se construye a partir de conceptos sólidos. Parece una serie de vasos comunicantes que se interconectan unos con otros formando redes de conocimientos. Allí, el discernimiento establece niveles cognoscitivos de comprensión e interpretación de la realidad concreta; por esta razón, lo que interesa es cómo los conocimientos nuevos se integran a los preexistentes y estos a la estructura cognitiva del sujeto. El propósito es que, estos conocimientos, perduren en el tiempo. 
En esta misma línea, lo que hace que el aprendizaje de Ausubel trascienda las fronteras de la enseñanza, es proporcionar un marco conceptual que desarrolla destrezas metacognitivas, las cuales están enfocadas en organizar los procesos cognitivos del educando (Ausubel, 1983). Aprendizaje que, además, se fundamenta en la experiencia previa, como lo explica el mismo autor: "El factor más importante que influye en el aprendizaje es lo que el alumno ya sabe" (Ausubel, 1983, p. 2) y tiene razón, porque el aprendizaje no puede empezar de cero.

Sin duda, para el aprendizaje significativo es importante que los conceptos, ideas, proposiciones pueden ser aprendidas, siempre y cuando, se establezcan relaciones con conceptos, ideas y las proposiciones preexistentes. De tal manera, la información es selecta cuando está adecuadamente "anclada" a la estructura cognitiva del individuo.

Como afirma David Ausubel (1983):

[...] el aprendizaje significativo ocurre cuando una nueva información "se conecta" con un concepto relevante "subsunsor" pre existente en la estructura cognitiva, esto implica que, las nuevas ideas, conceptos y proposiciones pueden ser aprendidos significativamente en la medida en que otras ideas, conceptos o proposiciones relevantes estén adecuadamente claras y disponibles en la estructura cognitiva del individuo y que funcionen como un punto de "anclaje" a las primeras (p. 14).

En otras palabras, los contenidos están relacionados de modo no arbitrario y sí sustancial con la estructura cognitiva del estudiante. Lo que explica que hay correspondencia entre lo que sabe y debe aprender el individuo. Ausubel, Novak y Hanesian (1983, p. 326) explican que "la esencia del aprendizaje significativo reside en el hecho de que las ideas están relacionadas simbólicamente y de manera no arbitraria (no al pie de la letra) con lo que el alumnado ya sabe", es decir, es una representación que se el resultado de la asociación de ideas que están conectadas y sistematizadas entre sí.

En el aprendizaje significativo, la nueva información interacciona e interactúa con los conocimientos (ideas, conceptos, relaciones) preexistentes del individuo para que sean vinculados al aprendizaje, por lo que los llamados "subsunsores" alimentan la estructura cognitiva de los estudiantes. De ahí que, los conocimientos crean puentes que atraviesan el proceso de asimilación para que la nueva información se convierta en un instrumento potencialmente significativo, para trascender en el sentido de aprender a aprender.

Finalmente, según Delors (1994) aprender cuando el estudiante "sabe conocer", es decir, comprende y fija o memoriza la información; pero también, cuando des-

1 Para Ausubel, la palabra subsunor se refiere a las estructuras y conocimientos previos que sirven de ancla o bases para la adquisición de nuevos conocimientos que, además, les dan sentido y son fundamentales para formar o sistematizar esos nuevos conocimientos. 
cubre su capacidad de reconocer "cómo hacer", poniendo en práctica sus conocimientos, experiencias y reflexiones al servicio de la sociedad. Finalmente, la idea es promover la asimilación de los conocimientos, por lo que, el docente utilizará organizadores previos que favorezcan la creación de relaciones adecuadas entre los saberes previos y los nuevos (Ausubel, 1983).

\section{Requerimientos del aprendizaje significativo}

Desde el punto de vista didáctico, el aprendizaje significativo tiene sus exigencias para que se cumpla el proceso de fijación y asimilación de los conocimientos. Las ventajas del aprendizaje significativo se centran en vincular la nueva información a la estructura cognitiva, superando las expectativas del memorístico, en el sentido de almacenar la información para desarrollar la memoria a corto y largo plazo, además el aprendizaje significativo es una forma de enseńanza cognitiva que interactúa directamente con las estructuras intelectuales de los individuos y, por último, también se relaciona con la motivación para ayudar a afirmar y apropiarse de los conocimientos.

En este contexto, una ventaja del aprendizaje significativo es que los nuevos conocimientos facilitan la retención de la nueva información en la estructura cognitiva; es decir, cumplen con la tarea de almacenar los nuevos conocimientos en la memoria a largo plazo. Según Carrillo-Mora (2010), este tipo de memoria es un grupo de funciones cerebrales que tienen la tarea de clasificar, codificar, almacenar y recuperar una gran diversidad de información que desarrolla el proceso aprendizaje-enseñanza.

A la par, el aprendizaje significativo es un proceso de enseñanza activa y personal, siempre que el estudiante aproveche las actividades y tareas de aprendizaje, e íntimo cuando depende de su equipaje cognoscitivo. De ahí que, las exigencias para lograr un aprendizaje significativo se circunscriben en los cuatro pilares de la educación: "aprender a conocer, aprender a hacer, aprender a vivir juntos y aprender a ser" (Delors, 1994, p. 8).

Igualmente, la motivación es otro factor notable en el aprendizaje significativo, pues brinda la oportunidad a los estudiantes de apropiarse con éxito de los conocimientos, a partir de desarrollar las habilidades y hábitos de estudios. Asimismo, es proceso psicológico que se basa en la afectividad. Según Alcalay y Antonijevic (1978), éste es un dispositivo que dirige la conducta hacia un logro, lo que implica variables: cognitivas, habilidades de pensamiento y conductas instrumentales para alcanzar metas. A la par, la motivación es la actitud -emocional y sociocultural-interna, que facilita la adquisición y retención de los nuevos conocimientos, por lo que, el papel del docente es fundamental cuando estimula, organiza, planifica actividades y tareas para que el estudiante desarrolle de manera intrapersonal y libre el proceso de enseńanza y aprendizaje.

Por otro lado, el aprendizaje significativo debe cumplir con otros requerimientos para desarrollar aprendizajes de calidad, como la utilización de un material poten- 
cialmente significativo y a la predisposición subjetiva para el aprendizaje. Situaciones que se circunscriben en tres obligaciones: significatividad lógica del material, significatividad psicológica del material y la actitud favorable del estudiante.

Para Ausubel (1983), la significatividad lógica del material se refiere a cómo están presentados y organizados los contenidos, a partir de una secuencia lógica y ordenada. En cambio, la significatividad psicológica del material se explica desde el argumento de cómo el alumno conecta los conocimientos previos con la nueva información, es decir, existe o no una estructura cognitiva sólida y fuerte, que le permita: primero asimilar y almacenar los conocimientos en la estructura cognitiva y, luego, desarrollar la memoria a corto y largo plazo. Finalmente, la actitud favorable del estudiante es un requisito necesario del aprendizaje significativo, de ahí que, la motivación es la disposición subjetiva para el aprendizaje, pues influye en el desempeño (esfuerzo y persistencia), mejorado las habilidades al momento de procesar de la información.

En este contexto, Ausubel (1983) plantea diferentes tipos de aprendizaje significativo, que pueden desarrollar etapas y características propias para desarrollar las habilidades y destrezas de aprendizaje. Así lo explica la tabla 1:

Tabla 1. Tipos de aprendizaje significativo

\begin{tabular}{ll}
\hline \multicolumn{1}{c}{ TIPO } & \multicolumn{1}{c}{ ETAPAS } \\
\hline $\begin{array}{l}\text { Aprendizaje de } \\
\text { representaciones }\end{array}$ & $\begin{array}{l}\text { Aprende palabras que representan } \\
\text { objetos reales que tienen significado } \\
\text { para él. Aunque no los identifica } \\
\text { como categorías }\end{array}$
\end{tabular}

Aprendizaje de conceptos

Aprendizaje de proposiciones

1. Por diferenciación progresiva
El niño comprende palabras que pueden usarse en diferentes contextos. Además, percibe conceptos abstractos

Conoce el significado de los conceptos

Cuando el concepto nuevo se subordina a conceptos más inclusores que el alumno ya conocía.

\section{CARACTERÍSTICAS}

El niño adquiere el vocabulario. Aprende la palabra "mamá" pero ésta sólo tiene significado para aplicarse a su propia madre

Comprende que la palabra "mamá" puede usarse también por otras personas refiriéndose a sus madres

Comprende conceptos abstractos como "gobierno", "país", "mamífero".

Puede formar frases que contengan dos o más conceptos en donde afirme o niegue algo

Conoce el concepto de triángulo y al conocer su clasificación puede afirmar: "Los triángulos pueden ser isósceles, equiláteros $\mathrm{o}$ escalenos". 
2. Por reconciliación integradora

3. Por combinación
Cuando el concepto nuevo es de mayor grado de inclusión que los conceptos que el alumno ya conocía
Conoce los perros, los gatos, las ballenas, los conejos y al conocer el concepto de "mamífero" puede afirmar: "Los perros, los gatos, las ballenas y los conejos son mamíferos".
Conoce los conceptos de rombo y cuadrado $y$ es capaz de identificar que: "El rombo tiene cuatro lados, como el cuadrado".

Fuente: Ausubel (1983)

\section{El aprendizaje memorístico o por descubrimiento}

Para Ausubel (2002), el aprendizaje memorístico o mecánico es un "continuum" del aprendizaje significativo y puede ser importante en ciertas etapas del conocimiento, como desarrollar algunas potencialidades intelectuales. Sin embargo, es un aprendizaje que carece de "subsunsores" apropiados que puedan relacionar la nueva información a la estructura cognitiva, es decir es almacenada arbitrariamente y no hay una interacción con los conocimientos preexistentes.

Un ejemplo es la memorización de las fórmulas físicas o matemáticas, las cuales se aprenden literalmente por simple asociación. En síntesis, ambos aprendizajes (el memorístico y significativo) son continuos y construyen una red de conocimientos sólidos y coherentes a largo plazo.

Según, Fermín M. González, F.C. Ibáńez, J. Casali, J.J. López y Joseph D. Movak (2000) citado por Ballester (2002), el aprendizaje basado en la repetición tiende a inhibir un nuevo aprendizaje, mientras que el aprendizaje significativo, facilita el nuevo aprendizaje relacionando la nueva información con los conocimientos previos. Por otra parte, los materiales aprendidos significativamente pueden ser retenidos durante un periodo relativamente largo de tiempo, mes es incluso ańos, mientras que la retención del conocimiento después de un aprendizaje memorístico por repetición mecánica es de un intervalo corto de tiempo medido en horas o días.

En esta misma línea, el aprendizaje memorístico a diferencia del significativo se caracteriza por su implicancia en el tiempo. Es un aprendizaje breve y a corto plazo que, a veces, imposibilita el nuevo aprendizaje (pensemos en la fase inicial de nuevos conocimientos), pues se hace difícil interactuar con los conocimientos previos, aunque pueden ser necesarios en algunos casos. Por tanto, es un aprendizaje que está destinado a cohabitar con la levedad. En cambio, el aprendizaje significativo facilita la adquisición de significados, así como la retención y la transferencia de lo aprendido (Ausubel, 2002). Su permanencia está sujeta al fortalecimiento, conservación 
y relevancia de los conocimientos que están vinculados a la estructura cognitiva de los individuos.

Afirma, Ausubel (2002, p. 2):

El aprendizaje mecánico puede ser necesario en algunos casos, por ejemplo, en la fase inicial de un nuevo cuerpo de conocimientos, cuando no existen conceptos relevantes con los cuales pueda interactuar, en todo caso el aprendizaje significativo debe ser preferido, pues, este facilita la adquisición de significados, la retención y la transferencia de lo aprendido.

En todo caso, el aprendizaje significativo exige la adquisición de significados lógicos, la retención de los conocimientos y la trasferencia de lo aprendido, por lo que la nueva información debe tener suficientes "subsunsores" para que se relacionen con la estructura cognitiva del estudiante.

Para Ballester (2002), Ausubel plantea:

[...] que el aprendizaje significativo da sentido a aquello que aprende y puede comprender el alumno, pues existen elementos de anclaje en la experiencia propia de los conceptos nuevos que se presentan de manera coherente e interconectada. El aprendizaje es por tanto un proceso de construcción individual y personal, los humanos integramos dentro de las estructuras de conocimiento aquellos conceptos que tienen en cuenta y se relacionan con lo que ya s abemos (p. 18).

Es decir, es un proyecto personal propio del ser humano, que lo prepara para la vida, integrando los nuevos conocimientos a la estructura cognoscitiva, para luego relacionarlos con lo que ya sabe.

Igualmente podemos relacionar el aprendizaje por descubrimiento y el aprendizaje significativo. Ambos unidos por la trascendencia del constructivismo que, a diferencia del conductismo, le interesa la forma cómo el individuo incorpora la información a su estructura cognitiva. Así el descubrimiento responde a un tipo de estrategia que sigue el docente, estimulando el pensamiento simbólico, la creatividad y la memoria del estudiante.

De esta manera, el aprendizaje por descubrimiento impulsa la capacidad innovadora del estudiante, que está asociada a la comprensión e interpretación de la información; por esta razón, supera las limitaciones de la enseñanza mecanicista y reductora (memorística), intentando siempre revelar suposiciones, que luego van a ser confirmadas sistemáticamente. Además, es un aprendizaje que potencia las estrategias propuestas por el docente y, a la vez, exalta las diferencias individuales del estudiante, desarrollando el proceso "aprender a aprender" (Delors, 1994, p. 91) para que el discente desarrolle su curiosidad y eleve la autoestima. Díaz-Barriga y Hernández (2002) consideran:

[...] que el aprendizaje por descubrimiento no debe ser presentado como opuesto al aprendizaje por exposición (recepción), ya que éste puede ser igual de eficaz, si se cumplen unas características. Así, el aprendizaje escolar puede darse por recepción o por descubrimiento y puede lograr un aprendizaje significativo (p. 225). 
Por otro lado, ambos aprendizajes (recepción y descubrimiento) solo pueden desarrollar un aprendizaje significativo, cuando los alumnos cumplen con procesos de aprendizajes y establecen relaciones con los conocimientos anteriores, es decir, es un aprendizaje que trasciende en el tiempo, siempre que los estudiantes logren crear y poner en práctica estrategias metodológicas adecuadas, para desarrollar sus propios aprendizajes.

Asimismo, Ausubel señala que "el aprendizaje memorístico o por recepción va perdiendo importancia gradualmente a medida que el niño adquiere más conocimientos, ya que al aumentar éstos se facilita el establecimiento de relaciones significativas con cualquier material" (Ausubel 1983 citado por Pozo, 2010, p. 212). En otras palabras, es la adquisición correcta de información, en que la participación del estudiante consiste en internalizar a corto plazo dicha información

A diferencia, el aprendizaje por descubrimiento potencia la creatividad y el pensamiento crítico, asegurando la conservación de la información. "El aprendizaje por recepción, si bien es fenomenológicamente más sencillo que el aprendizaje por descubrimiento surge paradójicamente ya muy avanzado el desarrollo y especialmente en sus formas verbales más puras logradas, implica un nivel mayor de madurez cognoscitiva" (Ausubel, 1983, p. 36). En síntesis, el aprendizaje puede darse por recepción o por descubrimiento, siempre y cuando cumplan la función estratégica de enseñar, produciendo aprendizajes de calidad, a lo que Ausubel los llama significativos.

Finalmente, debe quedar claro que, el aprendizaje significativo depende de los conocimientos previos, para que la nueva información sea almacenada en la estructura cognitiva; aprendemos los conceptos a través de redes conceptuales/mapas conceptuales. En otras palabras, el aprendizaje significativo se opone al memorístico, porque vincula los conceptos que aprendemos con los que ya habíamos aprendido (Ausubel, 1983). Así, lo explica la tabla 2:

Tabla 2.Aprendizaje significativo y memorístico

\begin{tabular}{|l|l|}
\hline APRENDIZAJE SIGNIFICATIVO & APRENDIZAJE MEMORÍSTICO \\
\hline $\begin{array}{l}\text { Los nuevos conocimientos se incorporan en } \\
\text { forma sustantiva en la estructura cognitiva del } \\
\text { alumno. }\end{array}$ & $\begin{array}{l}\text { Los nuevos conocimientos se incorporan en forma } \\
\text { arbitraria en la estructura cognitiva del alumno. }\end{array}$ \\
\hline $\begin{array}{l}\text { El alumno relaciona deliberadamente los } \\
\text { nuevos conocimientos con sus conocimientos } \\
\text { previos }\end{array}$ & $\begin{array}{l}\text { El alumno no realiza un esfuerzo para integrar } \\
\text { los nuevos conocimientos con sus conocimientos } \\
\text { previos. }\end{array}$ \\
\hline $\begin{array}{l}\text { El alumno quiere aprender aquello que se le } \\
\text { presenta porque lo considera valioso. }\end{array}$ & $\begin{array}{l}\text { El alumno no quiere aprender, pues no concede } \\
\text { valor a los contenidos presentados por el profesor }\end{array}$ \\
\hline
\end{tabular}

Fuente: Ausubel (1983) 


\section{El aprendizaje significativo y el modelo de Honey y Mumford}

Según Ausubel (2002), el aprendizaje significativo es un proceso cognitivo que desarrolla nuevos conocimientos, para que, sean incorporados a la estructura cognitiva del estudiante, conocimientos que solo pueden surgir si los contenidos tienen un significado, que los relacione con los anteriores, facilitando la interacción y restructuración de la nueva información con la prexistente.

De esta forma, el docente pude organizar estrategias didácticas (tareas y actividades), para que el estudiante construya sus propios conocimientos, lo importante es que -el discente- comprenda la nueva información, activando su memoria corto y largo plazo, pues "la memoria como proceso de aprendizaje tiene un carácter activo que consiste en recrear el significado haciéndolo propio" (Martínez, S., 1994, p. 10). En este contexto, si el docente conoce los estilos de aprendizaje de sus estudiantes, le permitirá planear procesos metodológicos, que provoquen un aprendizaje significativo trascendente.

Dunn y Dunn (1978); Keefe (1988); Guild y Garger (1998), y Alonso, Gallego y Honey (1994) definen a los estilos de aprendizaje como las características o rasgos cognitivos, afectivos y fisiológicos estables, expresados a través de la interacción de la conducta y personalidad de alguien, cuando realiza una tarea didáctica. Lo que implica que existen relaciones directas entre los estilos de aprendizaje y aprendizaje significativo, pues ambos involucran estrategias didácticas de reflexión y análisis.

Bajo este criterio, Rodríguez Cepeda (2018) explica, que no existe una sola manera de aprender, sino que las personas aprenden cuando desarrollan ciertas preferencias y diferencias individuales. Es así como, varios investigadores han desarrollado propuestas teóricas sobre estilos de aprendizaje como el modelo de Kolb (1974) y Honey y Mumford (1986). El primero, se basa en un aprendizaje basado en la experiencia de la vida: entre más se desarrollan las destrezas y habilidades, más conocimientos se asimilan (Freedman y Stumof, 1980). Mientras, el segundo, le interesa las preferencias individuales de aprendizaje: las personas aprenden según sus gustos, afinidades e intereses particulares, contrayendo espacios de aproximación con sus propios estilos (Legorreta, 2000; Gallego y Nevot, 2008).

Es así como, Kolb, Rubín, MacIntyre, James, y Brignardello (1974) citado por Rodríguez Cepeda (2018) consideran que el aprendizaje está condicionado a la experiencia en cuatro momentos: experiencia concreta, observación reflexiva, conceptualización abstracta y experimentación activa. Este planteamiento revela dos momentos del aprendizaje: la percepción de la información: recibir, procesar e interpretar el contenido a partir de experiencias y conceptos y el procesamiento mismo de la información, cuando se relaciona con las experiencias activas (capacidad de análisis y síntesis), y la observación reflexiva (Vaquerizo y Orden, 2012).

Según Kolb, Rubin, y MacIntyre, (1977), Freedman y Stumpf, (1980) y Gómez, Oviedo, Gómez, y López, (2012), citados por Cepeda (2018), esto originó el modelo de cuatro cuadrantes, en el que se describen los estilos de aprendizaje. 


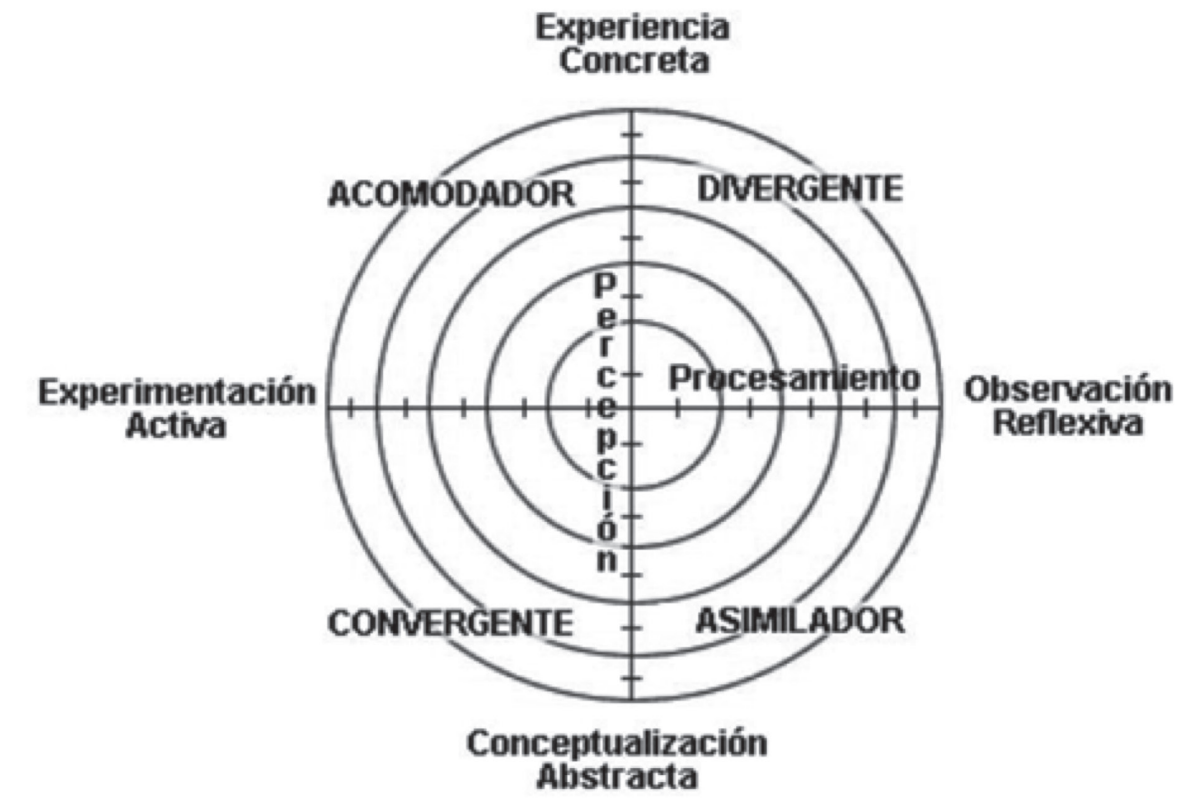

Figura 1. Matriz de los cuatro cuadrantes de los Estilos de aprendizaje de Kolb (Kolb, Rubin, y McIntyre, Psicología de las Organizaciones: Problemas Contemporáneos, 1977), citado por Cepeda (2018).

Como vemos, el modelo de Kolb es una experiencia circular del aprendizaje, que pone en evidencia las relaciones de aprendizaje, pues, las personas aprenden de diferente manera. Para Tulbure (2012), citado por Cepeda (2018), es necesario que el docente proponga estrategias de enseñanza basadas en los estilos de aprendizaje para mejorar los procesos de enseñanza y los discentes conozcan sus estilos de aprendizaje para mejorar su rendimiento para potenciar el aprendizaje autónomo.

Siguiendo a Honey y Mumford (1986), crean un cuestionario de estilos de aprendizaje LSQ (Learning Styles Questionaire), en el que proponen cuatro estilos de aprendizaje: activo, reflexivo, teórico y pragmático, más tarde acogido por Catalina Alonso (1992), quien lo adaptó al español con el nombre de CHAEA (Cuestionario Honey-Alonso sobre Estilos de Aprendizaje), basándose en los resultados obtenidos en su investigación, elaboró una lista con características que determinan el campo de destrezas de cada Estilo, como se explica en la tabla 3. 
Tabla 3. Características de los estilos de aprendizaje.

\begin{tabular}{|ll}
\hline ESTILOS & DESTREZAS \\
\hline Activo: & Animador, Improvisador, Descubridor, \\
& Arriesgado, Espontáneo \\
\hline Reflexivo & Ponderado, Concienzudo, Receptivo, Analítico, \\
& Exhaustivo \\
\hline Teórico & Metódico, Lógico, Objetivo, Crítico, Estructurado \\
\hline Pragmático & \\
\hline
\end{tabular}

Fuente: Alonso (1992)

Honey y Mumford (1986), como se dijo anteriormente, proponen un modelo de estilos de aprendizaje que está centrado en las preferencias individuales de los individuos: actitudes y comportamientos. Según Rodríguez Cepeda (2018), lo actitudinal y conductual están influenciados por factores externos propios de la globalización a los que los individuos están sometidos, por lo que es un proceso de aprendizaje de cuatro etapas, así se explica en la tabla 4:

Tabla 4. Factores externos.

\begin{tabular}{|ll|}
\hline Etapas aprendizaje & Características generales \\
\hline Experiencial & $\begin{array}{l}\text { El aprendizaje empieza cuando un individuo } \\
\text { tiene una experiencia acerca del objeto de } \\
\text { aprendizaje }\end{array}$ \\
\hline Revisión de la experiencia & $\begin{array}{l}\text { El individuo realiza un análisis de la } \\
\text { experiencia. }\end{array}$ \\
\hline Concluyendo desde la experiencia & Una vez analiza la experiencia elabora \\
conclusiones. & \\
\hline Planeación & $\begin{array}{l}\text { Con las conclusiones el individuo planea el } \\
\text { siguiente paso y retoma una experiencia, para } \\
\text { repetir nuevamente el ciclo. }\end{array}$ \\
\hline
\end{tabular}

Fuente: Honey y Mumford (1986) 
Para Legorreta (2000) citado por Rodríguez Cepeda (2018), manifiesta que, en la etapa de planeación, lo ideal sería que las personas puedan experimentar, reflexionar, formular conjeturas y aplicar, según sus preferencias individuales, que permitan a los estudiantes desarrollar estrategias de estudio, y a los docentes proponer estrategias de enseñanza que faciliten el aprendizaje significativo.

Es así como, el modelo de Honey y Mumford puede presentar combinaciones lógicas y de profunda significación; pues, busca, desde lo teórico, unir las estrategias didácticas con la manera particular que tienen los individuos para aprender, además profundiza en la relación de los estilos y las actividades planificadas (observación, discusión, reflexión, escucha, valoración), las cuales se juntan para provocar procesos de enseñanza-aprendizaje. Así lo explica la tabla 5.

Tabla 5. Características de los estilos de aprendizaje propuestos por Honey y Mumford.

\begin{tabular}{|c|c|c|c|}
\hline Estilo de aprendizaje & Características generales & $\begin{array}{l}\text { Actividades que les } \\
\text { favorece }\end{array}$ & $\begin{array}{c}\text { Actividades que no } \\
\text { les favorece }\end{array}$ \\
\hline Activo & $\begin{array}{l}\text { Aprenden haciendo } \\
\text { Necesita tener sus manos } \\
\text { ocupadas } \\
\text { Son de mente abierta } \\
\text { Se involucran en nuevas } \\
\text { experiencias. } \\
\text { Entusiastas } \\
\text { Actuan primero. } \\
\text { Consideran las consecuencias } \\
\text { después de actuar. } \\
\text { Les gusta rodearse de gente. }\end{array}$ & $\begin{array}{l}\text { Lluvia de ideas. } \\
\text { Solucionar problemas } \\
\text { Discusiones grupales. } \\
\text { Rompecabezas } \\
\text { Competición } \\
\text { Juego de roles. }\end{array}$ & $\begin{array}{l}\text { Adoptar un rol pasivo. } \\
\text { Trabajo independiente. } \\
\text { Actividades muy teóricas. }\end{array}$ \\
\hline Teórico & $\begin{array}{l}\text { Teorías antes de actuar } \\
\text { Necesitan modelos } \\
\text { Conceptos y hechos ordenados } \\
\text { Prefieren analizar y sintetizar. } \\
\text { Sistemáticos y lógicos } \\
\text { Perfeccionistas } \\
\text { Independiente } \\
\text { Analítico }\end{array}$ & $\begin{array}{l}\text { Elaborar modelos. } \\
\text { Estadísticas. } \\
\text { Buscar } \\
\text { antecedentes } \\
\text { Aplicar teorías. } \\
\text { Tener la oportunidad de indagar } \\
\text { y } \\
\text { preguntar }\end{array}$ & $\begin{array}{l}\text { Actividades ambiguas. } \\
\text { Actividades emocionales. } \\
\text { Actuar sin fundamento } \\
\text { teórico. }\end{array}$ \\
\hline Pragmático & $\begin{array}{l}\text { Prefieren la aplicación real de lo } \\
\text { aprendido. } \\
\text { No les gusta los conceptos } \\
\text { abstractos. } \\
\text { Les gusta probar nuevas ideas } \\
\text { aplicables a la vida real. } \\
\text { Su filosofia es: si funciona es } \\
\text { bueno }\end{array}$ & $\begin{array}{l}\text { Estudios de caso. } \\
\text { Con tiempo para pensar en } \\
\text { cómo aplicar lo aprendido. } \\
\text { Resolver problemas. } \\
\text { Discusiones. }\end{array}$ & $\begin{array}{l}\text { Actividades poco aplicables } \\
\text { a su realidad. } \\
\text { Actividades sin finalidad. } \\
\text { Actividades sin relación con } \\
\text { la realidad. }\end{array}$ \\
\hline Reflexivo & $\begin{array}{l}\text { Observadores } \\
\text { Analizan } \\
\text { Ven diversas perspectivas de } \\
\text { una sola cosa. } \\
\text { Prefieren tener un buen respaldo } \\
\text { antes de concluir algo. } \\
\text { Son cuidadosos para asegurar } \\
\text { algo. } \\
\text { Disfrutan observar y escuchar a } \\
\text { los demás. } \\
\text { Analizan las implicaciones. }\end{array}$ & $\begin{array}{l}\text { Discusiones pareadas. } \\
\text { Cuestionarios de autoanálisis. } \\
\text { Observando actividades. } \\
\text { Recibir retroalimentación de } \\
\text { otros. } \\
\text { Entrevistas. }\end{array}$ & $\begin{array}{l}\text { Presión del tiempo. } \\
\text { Actuar de líder. } \\
\text { Representar roles. } \\
\text { Actividades no planificadas. } \\
\text { Exponer ideas } \\
\text { espontáneamente. } \\
\text { Estar obligado a pasar de } \\
\text { una actividad a otra } \\
\text { rápidamente. }\end{array}$ \\
\hline
\end{tabular}

Fuente: Rodríguez Cepeda (2018) 
Según Gómez, Oviedo, Gómez y López (2012), no existe una sola forma de aprender, las personas aprenden según sus motivaciones y necesidades. De ahí que, los individuos busquen definir sus propios estilos de aprendizaje.

Finalmente, el docente debe integrar estrategias de aprendizaje y actividades planeadas, que faciliten la adquisición, almacenamiento y empleo de conocimientos, por lo que es necesario que conozcan las preferencias y diferencias individuales de sus estudiantes al momento de aprender. En este sentido, la estructura cognitiva del estudiante (que ha almacenado la información) está en sintonía con las preferencias individuales, lo que conlleva a un aprendizaje significativo.

\section{Conclusiones}

El aprendizaje significativo construye el conocimiento, a partir de esquemas que favorecen la retención a largo plazo. Sin embargo, adaptar estos organizadores a las estructuras cognitivas previas de los alumnos es difícil, por lo que la labor del docente es conocer los estilos de aprendizaje y preferencias individuales (modelo de Honey y Mumford) de sus alumnos para programar actividades y situaciones que conecten la estructura conceptual de una disciplina con la estructura cognitiva previa.

Sin duda, lo que hace que un aprendizaje sea significativo, es su trascendencia, al momento de construir el conocimiento en base a las experiencias previas, para que luego sea aplicado al mundo de la realidad, superando los límites de la inmanencia (en el sentido de quedarse o permanecer), porque es una actividad de la inteligencia que desarrolla e interioriza el conocimiento.

Por último, el aprendizaje significativo es un proceso activo e individual del ser humano que consiste en unir la nueva información con los conocimientos preexistentes del discente; por ello se debe considerar el material de apoyo potencialmente significativo. Es decir, los nuevos conocimientos se relacionan con la estructura cognitiva; por supuesto, según la disposición que tenga el estudiante para aprender. Por lo que el papel del docente es necesario cuando planifica las estrategias de aprendizaje, para facilitar el almacenamiento de conocimientos, que es el fin del aprendizaje significativo. En este sentido, la estructura cognitiva del estudiante debe relacionarse con los estilos de aprendizaje y las preferencias individuales, situación que provoca que el proceso enseñanza aprendizaje sea significativo.

\section{Referencias}

Alonso, C. (1992). Estilos de aprendizaje: análisis y diagnóstico en estudiantes universitarios. Madrid: Universidad Complutense.

Alonso, C., Gallego, D. y Honey, P. (1994). Los estilos de aprendizaje: procedimientos de diagnóstico y mejora. Bilbao: Ediciones Mensajero

Alcalay, L. y Antonijevic, N. (1987). Variables afectivas. Revista de Educación (México), 144, 29-32. 
Ausubel, D., Novak., J, D., y Hanesian, H. (1983). Psicología educativa: un punto de vista cognoscitivo. México: Trillas.

Ausubel. D. (2002). Adquisición y retención del conocimiento. Una perspectiva cognitiva. 2a edición, Barcelona: Paidós Ibérica.

Ayma, G. (1996). Curso: enseñanza de las ciencias: un enfoque Constructivista. Febrero UNSAAC.

Ballester, V. A (2002). El aprendizaje significativo en la práctica. Cómo hacer el aprendizaje significativo en el aula. Barcelona. Seminario de Aprendizaje Significativo.

Carrillo, Mora, P. (2010). Sistemas de memoria: reseña histórica, clasificación y conceptos actuales. Primera parte: Historia, taxonomía de la memoria, sistemas de memoria de largo plazo: la memoria semántica. Salud Mental. México, 33, $85-93$

Coll, Palacios, Marchesi (1992). Desarrollo psicológico y educación II. Madrid: Ed. Alianza.

Díaz, F. y Hernández, G. (2002). Estrategias docentes para un aprendizaje significativo. Una interpretación constructivista. 2da. edición, México: Editorial Mc Graw Hill.

Delors, Jacques (1994). "Los cuatro pilares de la educación”, en La Educación encierra un tesoro. El Correo de la UNESCO, México. pp. 91-103.

Dunn, R. y Dunn, K. (1978). Teaching Students throught their Individual Learning Styles: A practical aproach. New Jersey: Prentice Hall.

Fiszer, J. (S/F). ¿Aprendizaje significativo o aprendizaje memoristico? Recuperado en http://www.mental-gym.com/Docs/ARTICULO_101. pdf.

Freedman, R., \& Stumof, S. (1980). Learning Style Theory: Less than Meets The Eye. The Academy of Management Review, 5(3), 445-447.

Gallego, D., y Nevot, A. (2008). Los estilos de aprendizaje y la enseñanza de las matemáticas. Revista Complutense de Educación, 19(1), 95-112.

Gil, Pessoa (1992). Tendencias y experiencias innovadoras en la formación del profesorado de ciencias. Taller Subregional sobre Formación y Capacitación Docente. Caracas.

Gómez, L., Aduna, A., García, E., Cisneros, A., y Padilla, J. (2004). Manual de Estilos de Aprendizaje. México: Secretaría de Educación Pública.

Gómez, D., Oviedo, R., Gómez, A., y López, H. (2012). Estilos de aprendizaje en los estudiantes universitarios con base en el Modelo de Hemisferios Cerebrales. Revista Académica de Investigación, 1-23.

González, M. (2000). Evaluación del aprendizaje en la enseñanza universitaria. Revista Pedagogía Universitaria, (5) (2)

González, F.; Ibáńez, F.; Casali, J.; López, J. y Movak J, D. (2000). Una aportación a la mejora de la calidad de la docencia universitaria: los mapas conceptuales. Pamplona: Servicio de Publicaciones de la Universidad Pública de Navarra, p. 17.

Guild, P. y Garger, S. (1998). Marching to Different Drummers.USA: ASCDAsso- 
ciation for Supervision and Curriculum Development.

Honey, P. \& Mumford, A. (1986). The manual of learning styles. Maidenhead, Reino Unido: Berkshire.

Keefe, J. K. Profiling and utilizing learning styles. Virginia, 1988.

Kolb, D., Rubin, I., McIntyre, J., James, M., \& Brignardello, L. (1974). Psicología de las organizaciones: experiencias. México: Prentice Hall Hispanoamericana.

Legorreta, B. (2000). Fundamentos teórico-metodológicos de la educación a distancia: estilos de aprendizaje. Estado de Hidalgo: Universidad Autónoma del Estado de Hidalgo.

Martínez, S. (4 enero de 1994). La memoria y su relación con el aprendizaje. Sinéctica 4, 10. Recuperado de files.iramirez.webnode.es/200000012 38403393a7/ Memoria\%20y\%20aprendizaje.pdf

Méndez, R. (2006). Modelo de perfeccionamiento dirigido al mejoramiento de la gestión docente en el aula, basado en el constructivismo. Tesis doctoral. Universidad Santa María. [Tesis en línea]. Disponible en:http://www.monografias. $\mathrm{com} /$ trabajos40/gestion-docente/gestion-docente.sh tml. Consultado el 0505-2011.

Moreira, M.A. (1993). A teoría da aprendizagem significativa de David Ausubel. Fascículos de CIEF Universidad de Río Grande do Sul Sao Paulo.

Moreira M.A. (octubre de 1985) Metodología da pesquisa e metodología de encino: uma aplicaçao práctica. En: Ciencia e Cultura, 37(10).

Muñoz, B., y Silva, C. (September de 2003). Four Dimensions to Induce Learning: The Challenge Profile. (U. d. Navarra, Ed.) IESE Business School, 2-19.

Novak, J y Gowin, B. (1988). Aprendiendo a aprender. Barcelona: Edic. Martínez Roca.

Pozo, J. (2010). Teorías cognitivas del aprendizaje. Facultad de Psicología de la Universidad Autónoma de Madrid. Décima edición. Madrid, España: Ediciones Morata, S. L.Rodríguez Cepeda (2018) Los modelos de aprendizaje de Kolb, Honey y Mumford: implicaciones para la educación en ciencias. Sophia Revista de investigaciones en educación. (14) (1) 1-2

Rodríguez, M. (2004). La teoría del aprendizaje significativo. Centro de Educación a Distancia. España. [Artículo en línea]. Disponible en: http://cmc.ihmc.us/papers/cmc2004-290.pdf. Consultado el 04-05-2011.

Ortiz, N. (Lunes, 20 de septiembre de 2010). Mediación y Tecnología. Mediación de la trascendencia y del significado. Guatemala. Recuperado de http://manoteciii. blogspot.com/2010/09/resumen-mediacion-de-la-trascendencia-y.html

Palomino, Delgado, Valcarcel (1996). Enseñanza termo-dinámica: un enfoque Constructivista. II Encuentro de Físicos en la Región Inka. UNSAAC.Gil, Pessoa (1992). Tendencias y Experiencias Innovadoras en la Formación del Profesorado de Ciencias. Taller subregional sobre formación y capacitación docente. Caracas. Palomino, Delgado, Valcarcel (1996). Enseñanza termo-dinámica: un enfoque cons- 
tructivista. II Encuentro de Físicos en la Región Inka. UNSAAC.

Tulbure, C. (2012). Investigating the Relationships Between Teaching Strategies and Learning Styles in Higer Education. Acta Didáctica Napocensia, 5(4), 65-74.

Vaquerizo, Ma Belén y Orden, H., (10-13 de julio 2012). Experiencia de aprendizaje con metodologías activas y evaluación continua. Ciudad Real, 41-48. 